\title{
An improved approximation scheme for the centrifugal term and the Hulthén potential
}

\author{
Sameer M. Ikhdair ${ }^{1, *}$ \\ ${ }^{1}$ Department of Physics, Near East University, Nicosia, North Cyprus, Turkey
}

(Dated: December 16, 2018)

\begin{abstract}
We present a new approximation scheme for the centrifugal term to solve the Schrödinger equation with the Hulthén potential for any arbitrary $l$ state by means of a mathematical NikiforovUvarov (NU) method. We obtain the bound state energy eigenvalues and the normalized corresponding eigenfunctions expressed in terms of the Jacobi polynomials or hypergeometric functions for a particle exposed to this potential field. Our numerical results of the energy eigenvalues are found to be in high agreement with those results obtained by using the program based on a numerical integration procedure. The $s$-wave $(l=0)$ analytic solution for the binding energies and eigenfunctions of a particle are also calculated. The physical meaning of the approximate analytical solution is discussed. The present approximation scheme is systematic and accurate.

Keywords: Bound states, Hulthén potential, NU method, approximation schemes.
\end{abstract}

PACS numbers: 03.65.Ge, 12.39.Jh

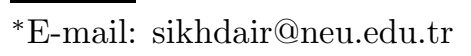




\section{INTRODUCTION}

It is necessary to obtain the exact bound state energy spectrum of the Schrödinger equations for some physical potential models. Therefore, much works have been done to solve the wave equation for various radial and angular potentials. Unfortunately, the exact analytic solutions (EAS) of idealized quantum systems (QS), under consideration, are possible only in the $s$-wave case with angular quantum number $l=0$ for some exponential-type potential models. On the other hand, the Schrödinger equation cannot be solved analytically

for $l \neq 0$ because of the centrifugal term potential $\frac{l(l+1)}{r^{2}}$. Over the past years, some authors [1-16] have used the approximation $\frac{l(l+1)}{r^{2}} \approx \frac{l(l+1) \delta^{2} e^{\delta r}}{\left(e^{\delta r}-1\right)^{2}}$ for the centrifugal term potential proposed by Greene and Aldrich [1] to obtain the $l \neq 0$ analytic bound-states $[2,4,5]$ and scattering states [7] solutions of the non-relativistic [2,5] and relativistic [6] wave equations with some exponential-type potentials such as Hulthén potential [2-7], Eckart potential [1013], Manning-Rosen potential [14-16] and diatomic molecular hyperbolical potential [17]. However, this approximation is valid only for small values of the screening parameter $\delta$ and it breaks down for large values of $\delta$ [5]. Therefore, there have been broad interest and impressive efforts in order to find a new approximation scheme which deals with the centrifugal term potential.

The Hulthén potential $[2,5,18]$ is the special case of the multiparameter exponential-type potential model $[19,20]$. It takes the form

$$
V(r)=-\frac{V_{0}}{e^{\delta r}-1}, V_{0}=Z e^{2} \delta
$$

where $V_{0}$ is a constant and $\delta$ is the screening parameter that determines the range of the potential. If the potential is used for atoms, then $V_{0}=Z \delta$ (in units $\hbar=c=e=1$ ), where $Z$ is identified as the atomic number. The Hulthén potential behaves like the Coulomb potential near the origin (i.e., $r \rightarrow 0) V_{C}(r)=-Z e^{2} / r$, but decreases exponentially in the asymptotic region when $r \gg 0$, so its capacity for bound states is smaller than the Coulomb potential [6,21-24]. This potential has been applied to a number of areas such as nuclear and particle physics [25-27], atomic physics [28-31], molecular physics [32,33] and chemical physics $[34,35]$, etc.

The bound-state EAS of the Schrödinger equation with the Hulthén potential can be solved in a closed form for $s$-waves (states with zero orbital angular momentum $l$ ) [36]. 
However, for the case $l \neq 0$, this quantum system cannot be exactly solved. For implementing approximate schemes economically and profitably; while dealing with practical quantum mechanical problems, EAS of the Hulthén potential is desirable although nonperturbative and numerical solutions of different potentials may lead to new physical ideas and/or calculational techniques in quantum physics. For instance, the numerical integration of the Schrödinger equation [37] is used to obtain the energy eigenvalues numerically for the Hulthén potential case, this provides a probe and/or test for the exactness of any analytic solution. One-parameter variational calculations are carried out in such numerical integration methods. The variational results are practically identical to the exact energies, except in the high-screening region. These variational calculations turn to become sophisticated in the solution of Schrödinger equation with multi-parameter potentials. However, no "exact" values obtained from a numerical integration of the Schrödinger equation have been available to assess the accuracy of the various methods [37]. Hence, it is important to note that the analytic solution of any quantum potential model, even if it is an approximated solution, is indispensable since the obtained expressions for energy eigenvalues and eigen functions contain all the necessary information regarding the quantum system under consideration.

In the non-relativistic case, for $l \neq 0$, several techniques have been used to obtain approximate analytic solutions, some authors have obtained the bound-state energy eigenvalues by using the numerical integration approach [37,38], quasi-analytical variational $[37,39]$, perturbation [40], SUSYQM [3], shifted 1/N expansion [41], AIM [5] and Nikiforov-Uvarov (NU) [2] methods. The results obtained by some of these methods [3,5] are in good agreement with the numerical integration approach [37] for low-screening region (small values of the screening parameter $\delta$ ) but the agreement becomes poor in the high-screening regime [5].

Recently, Haouat and Chetouani [42] have solved the Klein-Gordon and Dirac equations in the presence of the Hulthén potential, where the energy spectrum and the scattering wavefunctions are obtained for spin-0 and spin- $\frac{1}{2}$ particles, making a slight modification to the usual approximation scheme, $\frac{1}{r^{2}} \approx \alpha^{2} \frac{e^{-\gamma \alpha r}}{\left(1-e^{-\alpha r}\right)^{2}}$ where $\gamma$ is a dimensionless parameter $(\gamma=0,1$ and 2$)$ for the centrifugal term potential. They found that the good approximation, however, when the screening parameter $\alpha$ and the dimensionless parameter $\gamma$ are taken as $\alpha=0.1$ and $\gamma=1$, respectively, which is simply the case of the normal approximation used in the literature. Also, Jia and collaborators [43] have recently proposed a new alternative approximation scheme, $\frac{1}{r^{2}} \approx \alpha^{2}\left(\frac{\omega}{e^{\alpha r}-1}+\frac{1}{\left(e^{\alpha r}-1\right)^{2}}\right)$ where $\omega$ is a dimensionless parameter 
( $\omega=1.030$ ), for the centrifugal potential to improve the numerical energy eigenvalues of the Hulthén potential. When taking $\omega=1$, their approximation can be reduced to the usual approximation [1-16]. However, the accuracy of their numerical results [43] is still in poor agreement with the other numerical integration and variational methods [37] especially in high-screening $\delta$ regime. This problem could be solved by making a better approximation for the centrifugal term potential. In this work, for any arbitrary l-state, we aim to obtain approximate energy eigenvalues and corresponding normalized wave functions for the Hulthén potential in high agreement with the numerical method [37]. Hence, we present an alternative effective approximation that gives highly accurate numerical energy eigenvalues of the Hulthén potential as a function of screening parameter for all states with $Z=1$.

This paper is organized as follows: In the next Section, the NU method is briefly introduced. In Section 3, the l-states Schrödinger equation for the Hulthén potential is solved within the new effective approximation scheme and using the NU method. The calculated energy eigenvalues and wave functions are compared with the other ones found by using different analytical and numerical methods. The normalized wave functions are obtained in Section 4. Finally, the relevant conclusions are given in Section 5.

\section{NU METHOD}

The NU method is briefly introduced here and the details can be found in NikiforovUvarov handbook [44]. This method was proposed to solve the second-order differential wave equation of the hypergeometric-type:

$$
\psi_{n}^{\prime \prime}(s)+\frac{\widetilde{\tau}(s)}{\sigma(s)} \psi_{n}^{\prime}(s)+\frac{\widetilde{\sigma}(s)}{\sigma^{2}(s)} \psi_{n}(s)=0,
$$

where $\sigma(s)$ and $\tilde{\sigma}(s)$ are polynomials, at most of second-degree, and $\widetilde{\tau}(s)$ is a first-degree polynomial. The prime sign denotes the differentiation with respect to $s$. To find a particular solution of Eq. (2), one can decompose the wavefunction $\psi_{n}(z)$ as follows:

$$
\psi_{n}(s)=\phi_{n}(s) y_{n}(s)
$$

leading to a hypergeometric type equation

$$
\sigma(s) y_{n}^{\prime \prime}(s)+\tau(s) y_{n}^{\prime}(s)+\lambda y_{n}(s)=0
$$


The first part of the wavefunctions $\psi_{n}(s)$ is the solution of the differential equation,

$$
\sigma(s) \phi^{\prime}(s)-\pi(s) \phi(s)=0
$$

where

$$
\tau(s)=\widetilde{\tau}(s)+2 \pi(s)
$$

and $\lambda$ in (4) is a parameter defined as

$$
\lambda=\lambda_{n}=-n \tau^{\prime}(s)-\frac{n(n-1)}{2} \sigma^{\prime \prime}(s), n=0,1,2, \cdots .
$$

The $\tau(s)$ is a polynomial function of the parameter $s$ whose first derivative $\tau^{\prime}(s)$ must be negative which is the essential condition in choosing the proper solutions. The second part of the wavefunctions (3) is a hypergeometric-type function obtained by Rodrigues relation:

$$
y_{n}(s)=\frac{B_{n}}{\rho(s)} \frac{d^{n}}{d s^{n}}\left[\sigma^{n}(s) \rho(s)\right],
$$

where $B_{n}$ is a constant related to normalization and the weight function $\rho(s)$ can be found by $[44]$

$$
\sigma(s) \rho^{\prime}(s)+\left[\sigma^{\prime}(s)-\tau(s)\right] \rho(s)=0,
$$

The function $\pi(s)$ and the parameter $\lambda$ are defined as

$$
\begin{gathered}
\pi(s)=\frac{\sigma^{\prime}(s)-\widetilde{\tau}(s)}{2} \pm \sqrt{\left(\frac{\sigma^{\prime}(s)-\widetilde{\tau}(s)}{2}\right)^{2}-\tilde{\sigma}(s)+k \sigma(s)}, \\
\lambda=k+\pi^{\prime}(s),
\end{gathered}
$$

where $\pi(s)$ has to be a polynomial of degree at most one. The discriminant under the square root sign in Eq. (10) must be set to zero and then has to be solved for $k$ [44]. Finally, the energy eigenvalue equation is simply found by solving Eqs. (7) and (11).

\section{BOUND STATE SOLUTIONS}

The Schrödinger equation for a central molecular potential $V(r)$ can be written as

$$
\left(\frac{\hbar^{2}}{2 \mu} \nabla^{2}+E_{n l}-V(r)\right) \psi_{n l m}(\mathbf{r}, \theta, \phi)=0,
$$

where the representation of the Laplacian operator $\nabla^{2}$, in spherical coordinates, is

$$
\nabla^{2}=\frac{\partial^{2}}{\partial r^{2}}+\frac{2}{r} \frac{\partial}{\partial r}+\frac{1}{r^{2}}\left(\frac{1}{\sin \theta} \frac{\partial}{\partial \theta} \sin \theta \frac{\partial}{\partial \theta}+\frac{1}{\sin ^{2} \theta} \frac{\partial^{2}}{\partial \phi^{2}}\right)
$$


Here the wave functions $\psi_{n l m}(\mathbf{r}, \theta, \phi)$ belong to the energy eigenvalues $E_{n l}$ and $V(r)$ stands for the molecular potential in the configuration space and $r$ represents the three-dimensional intermolecular distance $\left(\sum_{i=1}^{3} x_{i}^{2}\right)^{1 / 2}$. Let us decompose the wave function in (12) as follows:

$$
\psi_{n l m}(\mathbf{r}, \theta, \phi)=r^{-1} u_{n l}(r) Y_{l m}(\theta, \phi)
$$

where $Y_{l m}(\theta, \phi)$ represents contribution from the hyperspherical harmonics that arise in higher dimensions. Substituting the wave functions (14) into Eq. (12), the result is $[45,46]$

$$
\left\{\frac{d^{2}}{d r^{2}}-\frac{l(l+1)}{r^{2}}+\frac{2 \mu}{\hbar^{2}}\left[E_{n l}-V(r)\right]\right\} u_{n, l}(r)=0,
$$

where $E_{n l}$ is the bound-state energy of the system under consideration, i.e., $E_{n l}<0$ and the term $\frac{l(l+1)}{r^{2}}$ is known as the centrifugal term. We also should be careful about the behavior of the wave function $u_{n l}(r)$ near $r=0$ and $r \rightarrow \infty$. Furthermore, $u_{n l}(r)$ should be normalizable [47].

We can rewrite Eq. (15) for the Hulthén potential as

$$
\frac{d^{2} u_{n l}(r)}{d r^{2}}+\left[\frac{2 \mu E_{n l}}{\hbar^{2}}+\frac{2 \mu Z e^{2} \delta}{\hbar^{2}} \frac{e^{-\delta r}}{1-e^{-\delta r}}-\frac{l(l+1)}{r^{2}}\right] u_{n l}(r)=0,
$$

where $E_{n l}$ is the bound state energy of the system and $n$ and $l$ signify the radial and angular quantum numbers, respectively. When $l=0$ ( $s$-wave), Eq. (16) with the Hulthén potential can be exactly solved [36,48-50], but for the case $l \neq 0$, Eq. (16) cannot be exactly solved. So we must find a new approximation to the entrifugal term to solve the equation analytically. The new proposed approximation is based on the expansion of the centrifugal term in a series of exponentials depending on the intermolecular distance $r$ and keeping terms up to second order. For small $0.4 \leq \delta r \leq 1.2$ [5] (i.e., small screening parameter $\delta$ ), Eq. (16) is very well approximated to centrifugal term. However, for large screening parameter, a better approximation to the centrifugal term should be made. Hence, instead of employing the usual approximation given in [1-16], we propose an alternative approximation scheme casted in the form:

$$
\begin{gathered}
\frac{1}{r^{2}} \approx \delta^{2}\left[d_{0}+v(r)+v^{2}(r)\right], v(r)=\frac{e^{-\delta r}}{1-e^{-\delta r}}, \\
\frac{1}{r^{2}} \approx \delta^{2}\left[d_{0}+\frac{1}{e^{\delta r}-1}+\frac{1}{\left(e^{\delta r}-1\right)^{2}}\right]
\end{gathered}
$$


for the centrifugal term which takes a similar ansätze like the Hulthén potential. Under the coordinate transformation $r \rightarrow x$, it is convenient to shift the origin by defining $x=$ $\left(r-r_{0}\right) / r_{0}$, we obtain

$$
(1+x)^{-2}=\gamma^{2}\left[d_{0}+\frac{1}{e^{\gamma(1+x)}-1}+\frac{1}{\left(e^{\gamma(1+x)}-1\right)^{2}}\right], \gamma=r_{0} \delta .
$$

Further, expanding Eq. (17) around $r=r_{0}(x=0)$, we obtain the following expansion:

$$
\begin{aligned}
& 1-2 x+O\left(x^{2}\right)=\gamma^{2}\left(d_{0}+\frac{1}{e^{\gamma}-1}+\frac{1}{\left(e^{\gamma}-1\right)^{2}}\right) \\
& -\gamma^{3}\left(\frac{1}{e^{\gamma}-1}+\frac{3}{\left(e^{\gamma}-1\right)^{2}}+\frac{2}{\left(e^{\gamma}-1\right)^{3}}\right) x+O\left(x^{2}\right),
\end{aligned}
$$

from which we have

$$
\begin{gathered}
\gamma^{2}\left[d_{0}+\frac{1}{e^{\gamma}-1}+\frac{1}{\left(e^{\gamma}-1\right)^{2}}\right]=1, \\
\gamma^{3}\left(\frac{1}{e^{\gamma}-1}+\frac{3}{\left(e^{\gamma}-1\right)^{2}}+\frac{2}{\left(e^{\gamma}-1\right)^{3}}\right)=2 .
\end{gathered}
$$

Therefore, the shifting parameter $d_{0}$ is to be found from the solution of the above two equations as

$$
d_{0}=\frac{1}{\gamma^{2}}-\frac{1}{e^{\gamma}-1}-\frac{1}{\left(e^{\gamma}-1\right)^{2}}=0.0823058167837972,
$$

where $e$ is the base of the natural logarithms, $e=2.718281828459045$ and the parameter $\gamma=0.4990429999$.

Therefore, we may cast the centrifugal term as

$$
\lim _{\delta \rightarrow 0} \delta^{2}\left[\frac{1}{\gamma^{2}}-\frac{1}{e^{\gamma}-1}-\frac{1}{\left(e^{\gamma}-1\right)^{2}}+\frac{e^{-\delta r}}{1-e^{-\delta r}}+\left(\frac{e^{-\delta r}}{1-e^{-\delta r}}\right)^{2}\right]=\frac{1}{r^{2}}
$$

To conclude, it is important to note that when $d_{0}=0$, the approximation expression (17) is reduced to the usual approximation used in literature [1-16]. The variation of the centrifugal term potential $l(l+1) / r^{2}$ and the proposed approximation expression given in (17) versus $\delta r$ are plotted in Figure 1. Obviously, the approximate centrifugal term potential (17) and $l(l+1) / r^{2}$ are similar and coincide in both high-screening as well as in the low-screening regimes as shown in Figure 1.

Inserting the approximation expression (17) into Eq. (16) and changing the variables $r \rightarrow s=e^{-\delta r}$ through the mapping function $s=f(r)$, where $r \in[0, \infty)$ or $s \in[1,0]$, leads us to obtain the following equation 


$$
u_{n l}^{\prime \prime}(s)+\frac{(1-s)}{s(1-s)} u_{n l}^{\prime}(s)+\frac{1}{[s(1-s)]^{2}}\left[-\varepsilon_{n l}^{2}+\left(c_{1}-c_{2}+2 \varepsilon_{n l}^{2}\right) s-\left(c_{1}+\varepsilon_{n l}^{2}\right) s^{2}\right] u_{n l}(s)=0
$$

where

$$
\varepsilon_{n l}=\sqrt{\Delta E_{l}-\frac{2 \mu E_{n l}}{\hbar^{2} \delta^{2}}}, \quad \Delta E_{l}=l(l+1) d_{0}, c_{1}=\frac{2 \mu Z e^{2}}{\hbar^{2} \delta}, c_{2}=l(l+1) .
$$

In the present work, we will deal with bound state solutions, i.e., the radial part of the wavefunction $\psi_{n l m}(\mathbf{r}, \theta, \phi)$ must satisfy the boundary condition that $u_{n l}(r) / r$ becomes zero when $r \rightarrow \infty$, and $u_{n l}(r) / r$ is finite at $r=0$. In addition, we require $E_{n l} \leq \frac{\hbar^{2} \delta^{2}}{2 \mu} \Delta E_{l}$, i.e., $\varepsilon_{n l} \geq 0[36,51-53]$. Comparing Eqs. (23) and (2), we obtain the relevant polynomials:

$$
\widetilde{\tau}(s)=1-s, \sigma(s)=s(1-s), \widetilde{\sigma}(r)=-\varepsilon_{n l}^{2}+\left(c_{1}-c_{2}+2 \varepsilon_{n l}^{2}\right) s-\left(c_{1}+\varepsilon_{n l}^{2}\right) s^{2} .
$$

Inserting the polynomials given by Eq. (25) into Eq. (10) gives the polynomial:

$$
\pi(s)=-\frac{s}{2} \pm \frac{1}{2} \sqrt{\widetilde{a} s^{2}+\widetilde{b} s+\widetilde{c}}
$$

where $\widetilde{a}=1+4\left(c_{1}+\varepsilon_{n l}^{2}-k\right), \widetilde{b}=4\left(k-c_{1}+c_{2}-2 \varepsilon_{n l}^{2}\right)$ and $\widetilde{c}=4 \varepsilon_{n l}^{2}$. The equation of quadratic form under the square root sign of Eq. (26) must be solved by setting the discriminant of this quadratic equal to zero: $\Delta=\widetilde{b}^{2}-4 \widetilde{a} \widetilde{c}=0$. This discriminant gives a new quadratic equation can be solved for the constant $k$ to obtain the two roots:

$$
k_{1,2}=c_{1}-c_{2} \pm \varepsilon_{n l} \sqrt{1+4 c_{2}}
$$

When the two values of $k$ given in Eq. (27) are substituted into Eq. (26), the four possible forms of $\pi(s)$ are obtained as

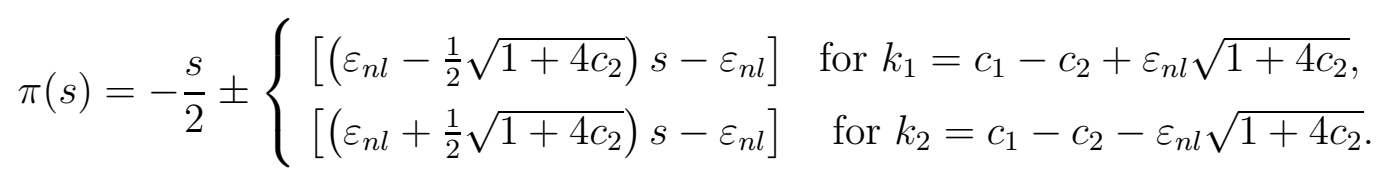

One of the four values of the polynomial $\pi(s)$ is just proper to obtain the bound state energy states because $\tau(s)$ given by Eq. (6) has a negative derivative for this value of $\pi(s)$ [44]. Therefore, the most suitable expression of $\pi(s)$ is chosen as

$$
\pi(s)=-\frac{s}{2}-\left[\left(\varepsilon_{n l}+\frac{1}{2} \sqrt{1+4 c_{2}}\right) s-\varepsilon_{n l}\right],
$$

for $k_{2}=c_{1}-c_{2}-\varepsilon_{n l} \sqrt{1+4 c_{2}}$. Hence, $\tau(s)$ and $\tau^{\prime}(s)$ are obtained

$$
\tau(s)=1+2 \varepsilon_{n l}-2\left[1+\varepsilon_{n l}+\frac{1}{2} \sqrt{1+4 c_{2}}\right] s,
$$




$$
\tau^{\prime}(s)=-2\left[1+\varepsilon_{n l}+\frac{1}{2} \sqrt{1+4 c_{2}}\right],
$$

where $\tau^{\prime}(s)$ represents the derivative of $\tau(s)$. Using Eqs. (25), (29) and (30), the following expressions for $\lambda$ and $\lambda_{n}$ are obtained, respectively,

$$
\begin{gathered}
\lambda=\lambda_{n}=n^{2}+\left[1+2 \varepsilon_{n l}+\sqrt{1+4 c_{2}}\right] n,(n=0,1,2, \cdots), \\
\lambda=c_{1}-c_{2}-\frac{1}{2}\left(1+2 \varepsilon_{n l}\right)\left[1+\sqrt{1+4 c_{2}}\right]
\end{gathered}
$$

where $n$ is the number of nodes of the radial wave function $u_{n, l}(r)$. When $\lambda=\lambda_{n}$, an expression for $\varepsilon_{n l}$ is obtained as

$$
\varepsilon_{n l}=\frac{c_{1}}{2(n+l+1)}-\frac{(n+l+1)}{2},(n, l=0,1,2, \cdots) .
$$

Also, with the aid of Eq. (24), the previous energy equation gives the following bound state energy eigenvalue equation:

$$
E_{n l}=\frac{\hbar^{2} \delta^{2}}{2 \mu}\left\{l(l+1) d_{0}-\left[\frac{\mu Z e^{2}}{\hbar^{2} \delta(n+l+1)}-\frac{(n+l+1)}{2}\right]^{2}\right\} .
$$

In the case of the $s$-wave $(l=0)$, the previous equation turns to be

$$
E_{n}=-\frac{\hbar^{2} \delta^{2}}{2 \mu}\left(\frac{\mu Z e^{2}}{\hbar^{2} \delta(n+1)}-\frac{n+1}{2}\right)^{2},
$$

which is identical to the ones obtained before using the factorization method [36], SUSYQM approach [3,28,54], quasi-linearization method [55] and NU method [2,6,32]. Further, if we take the shift parameter $d_{0}=0$ in the present approximation, Eq. (34) reduces to

$$
E_{n l}=-\frac{\hbar^{2} \delta^{2}}{2 \mu}\left[\frac{\mu Z e^{2}}{\hbar^{2} \delta(n+l+1)}-\frac{(n+l+1)}{2}\right]^{2},
$$

which is also identical with the energy eigenvalues formula given in Eq. (32) of Ref. [5], Eq. (24) of Ref. [43] and Eq. (28) of Ref. [2].

Let us turn to the calculations of the wave function $y_{n}(s)$, which is the first part solution of hypergeometric-type equation, we need to multiply Eq. (4) by the weight function $\rho(s)$ so that it can be rewritten in self-adjoint form [44]

$$
\left[\omega(s) y_{n}^{\prime}(s)\right]^{\prime}+\lambda \rho(s) y_{n}(s)=0
$$


The weight function $\rho(s)$ that satisfies Eqs. (9) and (37) is found as

$$
\rho(s)=s^{2 \varepsilon_{n l}}(1-s)^{(2 l+1)}
$$

which gives the Rodrigues relation (8):

$$
y_{n l}(s)=B_{n l} s^{-2 \varepsilon_{n l}}(1-s)^{-(2 l+1)} \frac{d^{n}}{d s^{n}}\left[s^{n+2 \varepsilon_{n l}}(1-s)^{n+2 l+1}\right]=B_{n l} P_{n}^{\left(2 \varepsilon_{n l}, 2 l+1\right)}(1-2 s) .
$$

Further, inserting the values of $\sigma(s)$ and $\pi(s)$ given in Eqs. (25) and (29) into Eq. (5), one can find the other part of the wave function as

$$
\phi(s)=s^{\varepsilon_{n l}}(1-s)^{(l+1)} .
$$

Hence, the wave functions in Eq. (3) become

$$
u_{n l}(s)=\mathcal{N}_{n l} s^{\varepsilon_{n l}}(1-s)^{l+1} P_{n}^{\left(2 \varepsilon_{n l}, 2 l+1\right)}(1-2 s), s \in[1,0)
$$

where $\mathcal{N}_{n l}$ is the normalization constant to be determined in the next section. Finally, the unnormalized radial wave functions are obtained as

$\psi_{n l m}(\mathbf{r}, \theta, \phi)=\mathcal{N}_{n l} r^{-1}\left(e^{-\delta r}\right)^{\varepsilon_{n l}}\left(1-e^{-\delta r}\right)_{2}^{l+1} F_{1}\left(-n, n+2\left(\varepsilon_{n l}+l+1\right) ; 2 \varepsilon_{n l}+1 ; e^{-\delta r}\right) Y_{l m}(\theta, \phi)$.

Thus, the Jacobi polynomials can be expressed in terms of the hypergeometric functions $[56]$ :

$$
P_{n}^{(a, b)}(1-2 x)={ }_{2} F_{1}(-n, n+a+b+1 ; a+1 ; x),
$$

where ${ }_{2} F_{1}(a, b ; c ; x)=\frac{\Gamma(c)}{\Gamma(a) \Gamma(b)} \sum_{k=0}^{\infty} \frac{\Gamma(a+k) \Gamma(b+k)}{\Gamma(c+k)} \frac{x^{k}}{k !}$. The hypergeometric function ${ }_{2} F_{1}(a, b ; c ; x)$ is a special case of the generalized hypergeometric function [56]

$$
{ }_{p} F_{q}\left(\alpha_{1}, \alpha_{2}, \cdots, \alpha_{p} ; \beta_{1}, \beta_{1}, \cdots, \beta_{q} ; x\right)=\sum_{k=0}^{\infty} \frac{\left(\alpha_{1}\right)_{k}\left(\alpha_{2}\right)_{k} \cdots\left(\alpha_{p}\right)}{\left(\beta_{1}\right)_{k}\left(\beta_{2}\right)_{k} \cdots\left(\beta_{q}\right)} \frac{x^{k}}{k !}
$$

where the Pochhammer symbol is defined by $(y)_{k}=\Gamma(y+k) / \Gamma(y)$.

In the case $l=0$, the above wave functions become

$$
\psi_{n}(\mathbf{r})=D_{n} r^{-1}\left(e^{-\delta r}\right)^{\varepsilon_{n}}\left(1-e^{-\delta r}\right)_{2} F_{1}\left(-n, n+2\left(\varepsilon_{n}+1\right) ; 2 \varepsilon_{n}+1 ; e^{-\delta r}\right),
$$

with $\varepsilon_{n}=\frac{\mu Z e^{2}}{\hbar^{2} \delta(n+1)}-\frac{n+1}{2}$ and $D_{n}$ is another normalization factor. This result is consistent with the NU method [2]. Further, if we take the shift parameter $d_{0}=0$ in the present 
approximation, Eq. (42) reduces to the form

$\psi_{n l m}(\mathbf{r}, \theta, \phi)=D_{n l} r^{-1}\left(e^{-\delta r}\right)^{\varepsilon_{n l}}\left(1-e^{-\delta r}\right)_{2}^{l+1} F_{1}\left(-n, n+2\left(\varepsilon_{n l}+l+1\right) ; 2 \varepsilon_{n l}+1 ; e^{-\delta r}\right) Y_{l m}(\theta, \phi)$,

with $\varepsilon_{n l}=\frac{\mu Z e^{2}}{\hbar^{2} \delta(n+l+1)}-\frac{n+l+1}{2}$ and $D_{n l}$ is a normalization factor. The critical screening $\delta_{c}=\frac{2 \mu Z e^{2}}{\hbar^{2}(n+l+1)^{2}}$ at which $E_{n l}=0$ has wave functions: $\psi_{n l m}(\mathbf{r}, \theta, \phi)=D_{n l} r^{-1}(1-$ $\left.e^{-\delta_{c} r}\right)^{l+1} P_{n}^{(0,2 l+1)}\left(1-2 e^{-\delta_{c} r}\right) Y_{l m}(\theta, \phi)$.

In order to show the accuracy of our analytical results, we present the numerical data in support of the results obtained in Eqs. (34) and (42) which are the main analytic results obtained in this work. Therefore, we calculate the energy eigenvalues for $Z=1, n$ and $l$ arbitrary quantum numbers as a function of the screening parameters $\delta$. The results calculated in Tables 1 and 2 by using Eq. (34) are compared with those obtained with the help of the numerical integration [37], asymptotic iteration [5], variational [37], SUSY [3] and the recently proposed approximation [43] methods. Tables 1 and 2 show that our results obtained with the new approximation scheme with the NU method are in high agreement with those obtained by numerical integration method [37] for short potential range (small screening parameter $\delta$ ). However, the slight differences in the energy eigenvalues from the numerical integration method [37] are observed for long potential range (large screening parameter $\delta)$. Therefore, our approximated numerical results are closer to the numerical integration results than the results obtained via AIM [5] using Eq. (32) and also the recently proposed approximation scheme [43] using Eq. (19) for small and large screening parameter $\delta$ values. Thus, the present approximation form (22) to the centrifugal term can highly improve the accuracy of calculating the energy eigenvalues for the Hulthén potential than the recently proposed approximation (5) given in [43].

\section{NORMALIZATION OF THE RADIAL WAVE FUNCTION}

Using $s(r)=e^{-\delta r}$ and Eq. (41), we are able to express normalization condition $\int_{0}^{\infty} u(r)^{2} d r=1$ as

$$
\frac{\mathcal{N}_{n l}^{2}}{\delta} \int_{0}^{1} s^{2 \varepsilon_{n l}-1}(1-s)^{2 l+2}\left[P_{n}^{\left(2 \varepsilon_{n l}, 2 l+1\right)}(1-2 s)\right]^{2} d s=1 .
$$

Unfortunately, there is no formula available to calculate this key integration. Neveretheless, we can find the explicit normalization constant $N_{n l}$. For this purpose, it is not difficult to 
obtain the results of the above integral by using the following formulas [56-59],

$$
P_{n}^{(\alpha, \beta)}(x)=(n+\alpha) !(n+\beta) ! \sum_{p=0}^{n} \frac{1}{p !(n+\alpha-p) !(\beta+p) !(n+p) !}\left(\frac{x-1}{2}\right)^{n-p}\left(\frac{x+1}{2}\right)^{p}
$$

and

$$
B(x, y)=\int_{0}^{1} t^{x-1}(1-t)^{y-1} d t=\frac{\Gamma(x) \Gamma(y)}{\Gamma(x+y)}, \operatorname{Re}(x), \operatorname{Re}(y)>0 .
$$

Thus, the normalization constant $\mathcal{N}_{n l}$ is now obtained as

$$
\mathcal{N}_{n l}=\frac{1}{(n+2 l+1) ! \Gamma\left(2 \varepsilon_{n l}+n+1\right)} \sqrt{\frac{\delta \Gamma\left(2 \varepsilon_{n l}+2 n+2 l+4\right)}{\Gamma\left(2 \varepsilon_{n l}+2 n+1\right) \sum_{p, q=0}^{n}\left(f_{p} f_{q} f_{p, q}\right)^{-1}}},
$$

where

$$
\begin{gathered}
f_{p}=(-1)^{p} p ! \Gamma\left(2 \varepsilon_{n l}+n-p+1\right)(2 l+p+1) !(n+p) ! \\
f_{q}=(-1)^{q} q ! \Gamma\left(2 \varepsilon_{n l}+n-q+1\right)(2 l+q+1) !(n+q) ! \\
f_{p, q}=(2 l+p+q+2) !
\end{gathered}
$$

\section{CONCLUSIONS}

In this work, we have proposed an alternative improved approximation scheme for the centrifugal term and used this approximation scheme together with the NU method to solve the Schrödinger equation with any orbital angular momentum number $l$ for the Hulthén potential. The bound state energy eigenvalues and the unnormalized radial wavefunctions have been calculated in analytical and numerical way. The analytic expressions for the energy eigenvalues and wavefunctions have been reduced to the $s$-wave case and the $d_{0}=0$ case (usual approximation) [1-16]. Our numerical results obtained by the approximation scheme given in expression (22) for the centrifugal term has been found to be more effective than the numerical results of the recently proposed approximation (5) of Ref. [43] and the commonly used approximation in generating the energy spectrum of the Hulthén potential. Our results in Tables 1 and 2 for small screening $\delta$ values show that the present approximation is in high agreement with the numerical integration and variational methods [37] whereas it is in quite good agreement for large screening $\delta$ values. The present approximation method is simple, practical and powerful than the other known methods $[2,5,43]$. This new method can be used 
for many quantum models to improve the accuracy of energy eigenvalues for few potential models of the exponential-type like the hyperbolical and Manning-Rosen potentials (cf. e.g., Refs. [60,61].)

\section{Acknowledgments}

The author thanks the kind referees for their useful suggestions. This work was partially supported by the Scientific and Technological Research Council (TÜBİTAK) of Turkey. 
[1] R.L. Greene and C. Aldrich, Phys. Rev. A 14 (1976) 2363.

[2] S.M. Ikhdair and R. Sever, J. Math. Chem. 42 (2007) 461.

[3] B. Gönül, O. Özer, Y. Cançelik and M. Kocak, Phys. Lett. A 275 (2000) 238; B. Gönül, Chin. Phys. Lett. 21 (2004) 1685.

[4] M. Aktaş and R. Sever, J. Mol. Struct. 710 (2004) 219.

[5] O. Bayrak, G. Kocak and I. Boztosun, J. Phys. A: Math. Gen. 39 (2006) 11521.

[6] S.M. Ikhdair, arXiv:0810.1590, to appear in Int. J. Mod. Phys. C 20 (1) (2009).

[7] C.-Y. Chen, F.-L. Lu and D.-S. Sun, Cent. Eur. J. Phys. 6 (2008) 884.

[8] U. Myhrman, J. Phys. A: Math. Gen. 16 (1983) 263.

[9] A. Bechlert and W. Bühring, J. Phys. B: At. Mol. Opt. Phys. 21 (1988) 817.

[10] S.H. Dong, W.C. Qiang, G.H. Sun and V.B. Bezerra, J. Phys. A: Math. Teeor. 40 (2007) 10535.

[11] G.F. Wei, C.Y. Long, X.Y. Duan and S.H. Dong, Phys. Scr. 77 (2008) 035001.

[12] C.Y. Chen, D.S. Sun and F.L. Lu, J. Phys. A: Math. Theor. 41 (2008) 035302.

[13] L.H. Zhang, X.P. Li and C.S. Jia, Phys. Lett. A 372 (2008) 2201.

[14] W.C. Qiang and S.H. Dong, Phys. Lett. A 368 (2007) 13.

[15] G.F. Wei, C.Y. Long and S.H. Dong, Phys. Lett. A 372 (2008) 2592.

[16] S.M. Ikhdair and R. Sever, Ann. phys. (Berlin) 17 (11) (2008) 897. .

[17] S.S. Dong, J. Garcia-Ravelo and S.H. Dong, Phys. Scr. 76 (2007) 393.

[18] L. Hulthén, Ark. Mat. Astron. Fys. A 28 (1942) 5.

[19] C.-S. Jia, X.-L. Zeng and L.-T. Sun, Phys. Lett. A 294 (2002) 185.

[20] C.-S. Jia, Y. Li, Y. Sun, J.-Y. Liu and L.-T. Sun, Phys. Lett. A 311 (2003) 115.

[21] S.M. Ikhdair and R. Sever, Int. J. Theor. Phys. 46 (2007) 1643.

[22] S.M. Ikhdair and R. Sever, Ann. Phys. (Leipzig) 16 (2007) 218.

[23] S.M. Ikhdair and R. Sever, Int. J. Mod. Phys. E 17 (6) (2008) 1107.

[24] M. Şimşek and H. Eğrifes, J. Phys. A: Math. Gen. 37 (2004) 4379.

[25] C.S. Lam and Y.P. Varshni, Phys. Rev. A 4 (1971) 1874.

[26] B. Durand and L. Durand, Phys. Rev. D 23 (1981) 1092.

[27] R.L. Hall, Phys. Rev. A 32 (1985) 14. 
[28] R. Dutt, K. Chowdhury and Y.P. Varshni, J. Phys. A: Math. Gen. 18 (1985) 1379; T. Xu, Z.Q. Cao, Y.C. Ou, Q.S. Shen and G.L. Zhu, Chin. Phys. 15 (2006) 1172.

[29] T. Tietz, J. Chem. Phys. 35 (1961) 1917; K. Szalcwicz and H.J. Mokhorst, J. Chem. Phys. 75 (1981) 5785.

[30] G. Malli, Chem. Phys. Lett. 26 (1981) 578.

[31] J. Lindhard and P.G. Hansen, Phys. Rev. Lett. 57 (1986) 965.

[32] I.S. Bitensky, V.K. Ferleger and I.A. Wojciechowski, Nucl. Instrum. Meth. B 125 (1997) 201.

[33] C.-S. Jia, J.Y. Wang, S. He and L.-T.Sun, J. Phys. A: Math. Gen. 33 (2000) 6993.

[34] P. Pyykko and J. Jokisaari, Chem. Phys. 10 (1975) 293.

[35] J.A. Olson and D.A. Micha, J. Chem. Phys. 68 (1978) 4352.

[36] S. Flügge, Practical Quantum Mechanics, Springer, Berlin, 1974.

[37] Y.P. Varshni, Phys. Rev. A 41 (1990) 4682.

[38] M.A. Nunez, Phys. Rev. A 47 (1993) 3620.

[39] S.H. Patil, J. Phys. A: Math. Gen. 34 (2001) 3153.

[40] P. Matthys and H.D. Meyer, Phys. Rev. A 38 (1988) 1168.

[41] A.Z. Tang and F.T. Chan, Phys. Rev. A 35 (1987) 911; B. Roy and R. Roychoudhury, J. Phys. A: Math. Gen. 20 (1987) 3051.

[42] S. Haouat and L. Chetouani, Phys. Scr. 77 (2008) 025005.

[43] C.-S. Jia, J.-Y. Liu and P.-Q. Wang, Phys. Lett. 372 (2008) 4779.

[44] A.F. Nikiforov and V.B. Uvarov, Special Functions of Mathematical Physics (Birkhauser, Bassel, 1988).

[45] S.M. Ikhdair and R. Sever, Z. Phys. C 56 (1992) 155; Z. Phys. C 58 (1993) 153; Z. Phys. D 28 (1993) 1; Hadronic J. 15 (1992) 389; Int. J. Mod. Phys. A 18 (2003) 4215; Int. J. Mod. Phys. A 19 (2004) 1771; Int. J. Mod. Phys. A 20 (2005) 4035; Int. J. Mod. Phys. A 20 (2005) 6509; Int. J. Mod. Phys. A 21 (2006) 2191; Int. J. Mod. Phys. A 21 (2006) 3989; Int. J. Mod. Phys. A 21 (2006) 6699; Int. J. Mod. Phys. E 17 (2008) 669.

[46] S.M. Ikhdair, Chin. J. Phys. 46 (2008) 291; S.M. Ikhdair and R. Sever, Int. J. Mod. Phys. 19 (2008) 1425; S.M. Ikhdair and R. Sever, Int. J. Mod. Phys. C 19 (2008) 221; Int. J. Mod. Phys. C 18 (2007) 1571; Cent. Eur. J. Phys. 5 (2007) 516; Cent. Eur. J. Phys. 6 (2008) 141; Cent. Eur. J. Phys. 6 (2008) 685; Cent. Eur. J. Phys. 6 (2008) 697; S.M. Ikhdair and R. Sever, DOI:10.1007/s10910-008-9438-8 to appear in J. Math. Chem. (2009). 
[47] G.T. Einevoll, P.C. Hemmer and J. Thomson, Phys. Rev. B 42 (1990) 3485.

[48] F. Dominguez-Adame, Phys. Lett. A 136 (1989) 175.

[49] L. Chetouani, L. Guechi, A. Lecheheb, T.F. Hammann and A. Messouber, Physics A 234 (1996) 529.

[50] B. Talukdar, A. Yunus and M.R. Amin, Phys. Lett. A 141 (1989) 326.

[51] H. Eğrifes and R. Sever, Int. J. Theor. Phys. 46 (2007) 935.

[52] G. Chen, Z.D. Chen and Z.M. Lou, Phys. Lett. A 331 (2004) 374.

[53] X.-C. Zhang, Q.-W. Liu, C.-S. Jia and L.-Z. Wang, Phys. Lett. A 340 (2005) 59.

[54] E.D Filho and R.M. Ricotta, Mod. Phys. Lett. A 10 (1995) 1613.

[55] V.B. Mandelzweig, Ann. Phys. 321 (2006) 2810.

[56] I.S. Gradshteyn and I.M Ryzhik, Tables of Integrals, Series, and Products, 5th edn (New York, Academic, 1994).

[57] G. Sezgo, Orthogonal Polynomials, (American Mathematical Society, New York, 1939).

[58] W. Magnus, F. Oberhettinger and R.P. Soni, Formulas and Theorems for the Special Function of Mathematical Physics, 3rd Ed., (Berlin, Springer, 1966).

[59] M. Abramowitz and I.A. Stegun, Handbook of Mathematical Functions (Dover, New York, 1964); A.P. Prudrinkov, Yu.A. Brychkov and O.I. Marichev, Integrals and Series, (New York, Gordon and Breach, 1986).

[60] S.M. Ikhdair and R. Sever, arXiv:0809.2485 submitted to Ann. Phys. (Berlin) (2008).

[61] S.M. Ikhdair and R. Sever, arXiv:0807.2085 submitted to Int. J. Mod. Phys. B (2008). 
FIG. 1: A plot of the variation of the centrifugal term, $1 / r^{2}$ and its corresponding approximation expression $\delta^{2}\left[d_{0}+\frac{e^{\delta r}}{\left(e^{\delta r}-1\right)^{2}}\right]$ versus $\delta r$, where the screening parameter $\delta$ changes from $\delta=0.050$ to $\delta=0.250$ in steps of 0.050 . The parameters are in atomic units $(\hbar=\mu=e=1)$ and $Z=1$. 
TABLE I: Bound energy spectra of the Hulthén potential as a function of the screening parameter $\delta$ for $2 p, 3 p$ and $3 d$ states for $Z=1$ in atomic units $\left(\hbar^{2}=\mu=e=1\right)$

\begin{tabular}{|c|c|c|c|c|c|c|c|}
\hline State & & Present $^{a}$ & Previous $[43]^{b}$ & Numerical [37] & AIM [5] & Variational 37 & SUSY [3] \\
\hline \multirow[t]{9}{*}{$2 p$} & 0.025 & 0.1127611 & 0.1126344 & 0.1127605 & 0.1128125 & 0.1127605 & 0.1127605 \\
\hline & 0.050 & 0.1010442 & 0.1009128 & 0.1010425 & 0.1012500 & 0.1010425 & 0.1010425 \\
\hline & 0.075 & 0.0898495 & 0.0898350 & 0.0898478 & 0.0903125 & 0.0898478 & 0.0898478 \\
\hline & 0.100 & 0.0791769 & 0.0794011 & 0.0791794 & 0.0800000 & 0.0791794 & 0.0791794 \\
\hline & 0.150 & 0.0593981 & 0.0604650 & 0.0594415 & 0.0612500 & 0.0594415 & 0.0594415 \\
\hline & 0.200 & 0.0417078 & 0.0441045 & 0.0418860 & 0.0450000 & 0.0418860 & 0.0418854 \\
\hline & 0.250 & 0.0261059 & 0.0303195 & 0.0266111 & 0.0312500 & 0.0266108 & 0.0266060 \\
\hline & 0.300 & 0.0125925 & 0.0191101 & 0.0137900 & 0.0200000 & 0.0137878 & 0.0137596 \\
\hline & 0.350 & 0.0011675 & 0.0104763 & 0.0037931 & 0.0112500 & 0.0037734 & 0.0036146 \\
\hline \multirow[t]{5}{*}{$3 p$} & 0.025 & 0.0437072 & 0.0436848 & 0.0437069 & 0.0437590 & 0.0437069 & 0.0437068 \\
\hline & 0.050 & 0.0331623 & 0.0332390 & 0.0331645 & 0.0333681 & 0.0331645 & 0.0331632 \\
\hline & 0.075 & 0.0239207 & 0.0242183 & 0.0239397 & 0.0243837 & 0.0239397 & 0.0239331 \\
\hline & 0.100 & 0.0159825 & 0.0166227 & 0.0160537 & 0.0168056 & 0.0160537 & 0.0160326 \\
\hline & 0.150 & 0.0040162 & 0.0057067 & 0.0044663 & 0.0058681 & 0.0044660 & 0.0043599 \\
\hline \multirow[t]{5}{*}{$3 d$} & 0.025 & 0.0436044 & 0.0435371 & 0.0436030 & 0.0437587 & 0.0436030 & 0.0436030 \\
\hline & 0.050 & 0.0327508 & 0.0329817 & 0.0327532 & 0.0333681 & 0.0327532 & 0.0327532 \\
\hline & 0.075 & 0.0229948 & 0.0238893 & 0.0230307 & 0.0243837 & 0.0230307 & 0.0230306 \\
\hline & 0.100 & 0.0143364 & 0.0162600 & 0.0144842 & 0.0168055 & 0.0144842 & 0.0144832 \\
\hline & 0.150 & 0.0003124 & 0.0053907 & 0.0013966 & 0.0058681 & 0.0013894 & 0.0132820 \\
\hline
\end{tabular}

${ }^{a}$ The present approximation.

${ }^{b}$ Jia et al approximation. 
TABLE II: Bound energy spectra of the Hulthén potential as a function of the screening parameter $\delta$ for $4 p, 4 d, 4 f, 5 p, 5 d, 5 f, 5 g, 6 p, 6 d, 6 f$ and $6 g$ states for $Z=1$ in atomic units $\left(\hbar^{2}=\mu=e=1\right)$

\begin{tabular}{|c|c|c|c|c|c|c|}
\hline State & Present $^{a}$ & Previous $[43]^{b}$ & Numerical [37] & ] AIM [5] & Variational [37] & SUSY [3] \\
\hline \multirow[t]{4}{*}{$4 p$} & 0.0250 .0199486 & 0.0199625 & 0.0199489 & 0.0200000 & 0.0199489 & 0.0199480 \\
\hline & 0.0500 .0110442 & 0.0111938 & 0.0110582 & 0.0112500 & 0.0110582 & 0.0110430 \\
\hline & 0.0750 .0045370 & 0.0049439 & 0.0046219 & 0.0050000 & 0.0046219 & 0.0045385 \\
\hline & 0.1000 .0004269 & 0.0012128 & 0.0007550 & 0.0012500 & 0.0007532 & 0.0004434 \\
\hline \multirow[t]{3}{*}{$4 d$} & 0.0250 .0198457 & 0.0198877 & 0.0198462 & 0.0200000 & 0.0198462 & 0.0198460 \\
\hline & $\begin{array}{ll}0.050 & 0.0106327\end{array}$ & 0.0110819 & 0.0106674 & 0.0112500 & 0.0106674 & 0.0106609 \\
\hline & 0.0750 .0036111 & 0.0048327 & 0.0038345 & 0.0050000 & 0.0038344 & 0.0037916 \\
\hline \multirow[t]{3}{*}{$4 f$} & $0.025 \quad 0.0196914$ & 0.0197756 & 0.0196911 & 0.0200000 & 0.0196911 & 0.0196911 \\
\hline & $\begin{array}{ll}0.050 & 0.0100154\end{array}$ & 0.0109150 & 0.0100620 & 0.0112500 & 0.0100620 & 0.0100618 \\
\hline & 0.0750 .0022222 & 0.0046682 & 0.0025563 & 0.0050000 & 0.0025557 & 0.0025468 \\
\hline \multirow[t]{2}{*}{$5 p$} & 0.0250 .0094017 & 0.0094325 & 0.0094036 & 0.0094531 & & 0.0094011 \\
\hline & $0.050 \quad 0.0026067$ & 0.0027900 & 0.0026490 & 0.0028125 & & 0.0026056 \\
\hline \multirow[t]{2}{*}{$5 d$} & 0.0250 .0092988 & 0.0093914 & 0.0093037 & 0.0094531 & & 0.0092977 \\
\hline & $0.050 \quad 0.0021952$ & 0.0027454 & 0.0023131 & 0.0028125 & & 0.0022044 \\
\hline \multirow[t]{2}{*}{$5 f$} & 0.0250 .0091445 & 0.0093898 & 0.0091521 & 0.0094531 & & 0.0091507 \\
\hline & $0.050 \quad 0.0015779$ & 0.0026791 & 0.0017835 & 0.0028125 & & 0.0017421 \\
\hline \multirow[t]{2}{*}{$5 g$} & 0.0250 .0089387 & 0.0092480 & 0.0089465 & 0.0094531 & & 0.0089465 \\
\hline & $0.050 \quad 0.0007549$ & 0.0025920 & 0.0010159 & 0.0028125 & & 0.0010664 \\
\hline $6 p$ & 0.0250 .0041500 & 0.0041899 & 0.0041548 & 0.0042014 & & 0.0041493 \\
\hline $6 d$ & 0.0250 .0040471 & 0.0041671 & 0.0040606 & 0.0042014 & & 0.0040452 \\
\hline $6 f$ & $\begin{array}{ll}0.025 & 0.0038927\end{array}$ & 0.0042014 & 0.0039168 & 0.0042014 & & 0.0038901 \\
\hline $6 g$ & 0.0250 .0036870 & 0.0040876 & 0.0037201 & 0.0042014 & & 0.0036943 \\
\hline
\end{tabular}

${ }^{a}$ The present approximation.

${ }^{b}$ Jia et al approximation. 